\title{
The molecular basis of loss of smell in 2019-nCoV infected individuals
}

Krishan Gupta ${ }^{1, *}$, Sanjay Kumar Mohanty ${ }^{5, *}$, Siddhant Kalra ${ }^{5, *}$, Aayushi Mittal ${ }^{5}$, Tripti Mishra $^{3}$, Jatin Ahuja ${ }^{2}$, Debarka Sengupta ${ }^{1,4,5, \#}$, and Gaurav Ahuja ${ }^{5, \#}$

${ }^{1}$ Department of Computer Science and Engineering, Indraprastha Institute of Information Technology-Delhi (IIIT-Delhi), Okhla, Phase III, New Delhi-110020, India ${ }^{2}$ Max Super Speciality Hospital, Press Enclave Marg, Saket Institutional Area, Saket, New Delhi, Delhi 110017Saket

${ }^{3}$ Pathfinder Research and Training Foundation, 30/7 and 8, Knowledge Park III, Greater Noida, Uttar Pradesh - 201308, India

${ }^{4}$ Infosys Center for Artificial Intelligence, Indraprastha Institute of Information Technology, Delhi 110020, India

${ }^{5}$ Department of Computational Biology, Indraprastha Institute of Information Technology-Delhi (IIIT-Delhi), Okhla, Phase III, New Delhi-110020, India

${ }^{*}$ These authors contributed equally $\quad{ }^{\#}$ Co-corresponding authors Additional Information:

To whom correspondence should be addressed. Gaurav Ahuja; Tel: (+91)11-26907475; Email: gaurav.ahuja@iiitd.ac.in. Correspondence may also be addressed to Debarka Sengupta; Tel: (+91)11-26907446; Email: debarka@iiitd.ac.in

The Ahuja lab is supported by the Ramalingaswami Re-entry Fellowship, a re-entry scheme of the Department of Biotechnology (DBT), Ministry of Science \& Technology, Govt. of India and an intramural Start-up grant from Indraprastha Institute of Information Technology-Delhi (IIIT-Delhi) and The Sengupta lab is funded by the INSPIRE faculty grant from the Department of Science \& Technology (DST), India.

The authors declare that they have no competing interests.

Keywords: Smell, COVID-19, Pandemic, SARS-COV-2, Olfaction, Olfactory sensory neurons (OSNs) 


\section{Key Points}

- Loss of smell (Anosmia) is now emerging as one of the key early symptoms in 2019-nCoV infected individuals worldwide, however, the identity of the cell types responsible for this striking phenotype is still elusive.

- We report here that the loss of smell in the 2019-nCoV infected patients may be due to the direct impact on sustentacular cells (supporting cells), olfactory stem cells and Bowman's gland cells of the olfactory epithelium.

- COVID-19 specifically targets key cell types responsible for olfactory tissue homeostasis, therefore explains the sudden loss/changes in smell phenotype.

- COVID-19 does not infect the olfactory sensory neurons (OSNs).

- Other mammalian species possess an equally potential risk to develop COVID-19 induced loss of smell phenotype, and therefore, could also serve as potential infection carriers. 


\section{Authors description:}

1. Krishan Gupta is a Ph.D. student of Indraprastha Institute of Information Technology, Delhi. His research interests include developing methods for the single-cell RNA sequencing data analysis.

2. Sanjay Kumar Mohanty is a student of Master's in Bioinformatics, presently working on his thesis dissertation at Indraprastha Institute of Information Technology, Delhi.

3. Siddhant Kalra is an M-Tech student (Master of Technology in Computational Biology) at the Indraprastha Institute of Information Technology, Delhi. His research interests include olfaction and computational neuroscience.

4. Aayushi Mittal is a Ph.D. student at the Indraprastha Institute of Information Technology, Delhi. Her research interests include gene expression regulation and epigenetics.

5. Tripti Mishra Ph.D. is a data scientist at the Pathfinder Research and Training Foundation.

6. Jatin Ahuja DNB, DM is a clinician with super-specialization (Doctorate of Medicine) in Infection Diseases.

7. Debarka Sengupta Ph.D. is a Professor at the Department of Computational Biology, Department of Computer Science at Indraprastha Institute of Information Technology. His research interests include single-cell genomics, liquid biopsy, and machine learning.

8. Gaurav Ahuja Ph.D. is an Assistant Professor at the Department of Computational Biology at the Indraprastha Institute of Information Technology-Delhi (IIIT-Delhi). His research interests include decoding the gene regulatory mechanisms mediating diseases onset/progression, cell-fate transitions, and cellular response to ligands. 


\begin{abstract}
Among the prominent clinical symptoms such as fatigue, shortness of breath, fever, and cough, 2019-nCoV infected individuals often experience hyposmia/anosmia (decrease or loss of sense of smell). Angiotensin I Converting Enzyme 2 (ACE2), a key host receptor has now been established as an important moiety for the entry of 2019-nCoV into the host cells. A multitude of studies estimated the expression of ACE2 in multiple organs including heart, kidney, intestine, lungs, buccal cavity, etc. The ongoing medical examinations and the autopsy reports of the diseased individuals strongly corroborate these organ/tissue-level molecular insights. Olfactory mucosa harbors multiple functionally distinct cell types. Zeroing in on the cell lineages that underpin infection associated loss of olfaction may provide new leads for diagnostics/clinical management of 2019-nCoV infected individuals. Our pointed bioinformatic analysis of single-cell expression profiles underscored selective expression of ACE2 in a subset of sustentacular cells (SUSs), Olfactory Stem cells (HBCs and GBCs), and Bowman's gland cells of the olfactory mucosa in humans. Co-expression analysis of ACE2 and TMPRSS2 (protease), two host-specific moieties indispensable for 2019-nCoV entry into the host cell revealed the highest infection susceptibility for the sustentacular cells. Additionally, an inspection of the ACE2 and TMPRSS2 levels in the olfactory mucosa of 4 additional mammalian species revealed comparable expression patterns, indicating the potential risk of olfactory dysfunction in these species. In summary, our findings pinpoint the molecular rationale of loss of smell in 2019-nCoV infected patients.
\end{abstract}

\title{
Introduction
}

The recent outbreak of the novel coronavirus 2019-nCoV triggered the urgent requirement of diagnostic methods, which can be rapidly deployed and therefore, timely employed by masses across the world [1-4]. In pursuit of this, various workgroups have extensively generated, curated and analyzed virus-centric datasets $[5,6]$. Some major efforts include virus isolation from the airway epithelial cells and its genome sequencing [5-7]. Comparative genomics revealed that 2019-nCoV is closely related to bat 
SARS-like coronaviruses (bat-SL-CoVZC45 and bat-SLCoVZXC21) [5]. Notably, the external subdomain of Spike's Receptor-Binding Domain (RBD) of 2019-nCoV shares $\sim 40 \%$ identity at the amino acid level with other SARS-related coronaviruses [8]. Of note, most of the amino acid differences of the RBD are located in the external subdomain, which is responsible for the direct interaction with the host receptors. Further, reports indicate the role of angiotensin-converting enzyme II (ACE2) as a prominent surface receptor for the cellular entry of 2019-nCoV $[9,10]$. Mechanistic insights further revealed the involvement of viral S-protein in assisting strong interaction with the host ACE2 receptor [11]. All these studies collectively reinforce the involvement of ACE2 in the viral entry into the host cell. In order to determine the tissue or organ level impact of 2019-nCoV, various groups have traced ACE2 expression in multiple organs/cell-types [12-15]. Notably, many of these studies have leveraged single-cell sequencing technology to pin-point the cell subpopulation of interest. Collectively, higher ACE2 expression was observed in a range of tissue/cell-types such as epithelial cells of the esophagus, absorptive enterocytes of the intestines, mucosal cells of the oral cavity, proximal tubule cells of the kidney, myocardial cells of the heart, urothelial cells of the bladder, etc, thereby making them potentially vulnerable to the 2019-nCoV infection [12-15]. Moreover, in addition to the ACE2 protein of the host cellular surface, TMPRSS2, a cell surface protease also emerged as a crucial host-specific molecule for the cellular entry of 2019-nCoV [16]. Mechanistically, recent reports indicate that the proteolytic activity of TMPRSS2 protease facilitates the cleavage of $S$ protein, a prerequisite for the viral-host interaction [16]. All these molecular findings are largely in line with the clinical symptoms reported worldwide, in which multi-organ failure is emerging as a major contributor to the infection-associated mortality.

We leveraged some recently published high-throughput single-cell expression studies to re-evaluate ACE2 expression levels among cell types of the olfactory mucosa. Our analysis revealed selective expression ACE2 in a subset of HBCs and SUSs. Notably, marginal expression was observed in a subset of Bowman's gland and globular basal 
cells, whereas no expression was observed in other cell types such as olfactory ensheathing glia, microvillar cells, immature or mature olfactory sensory neurons.

\section{Results}

\section{Predominant and selective expression of ACE2 and TMPRSS2 in a subpopulation of olfactory cells.}

We evaluated the expression of $A C E 2$ transcript in 3906 olfactory mucosa originated single cells from the recent report by Durante and colleagues [17], collectively entailing eight distinct olfactory cell types namely horizontal basal cells, microvillar cells, Bowman's gland cells, globular basal cells, olfactory ensheathing glia, sustentacular cells, immature and mature olfactory sensory neurons. We performed unsupervised clustering of the individual cells using the Seurat software suite [18]. Clusters were unambiguously mapped to specific cell types based on previously known markers (Fig. 1A; FigS1,2). Our analysis revealed that the expression of ACE2 is restricted to a subset of sustentacular and horizontal basal cells of the olfactory mucosa, collectively comprising less than $1 \%$ of the total analyzed cell population (Fig. 1B). Notably, a minor sub-fraction of the globular basal cells and Bowman's gland cells also exhibited ACE2 expression (Fig. 1C). To determine if the $A C E 2$-positive $\left(A C E 2^{+}\right)$subpopulation of HBCs and SUSs are indeed functionally distinct from the ACE-negative (ACE2) cells, we performed differential expression analysis between two subgroups of the horizontal basal cells ( $\mathrm{HBC}^{+} ; \mathrm{ACE}^{+}$and $\mathrm{HBC}^{+}$: $\left.\mathrm{ACE} 2^{-}\right)$, segregated based on $\mathrm{ACE} 2$ expression. Gene ontologies referring to the biological processes revealed significant distinction among the subpopulations of horizontal basal cells (Fig. 1D). Similar results were obtained for sustentacular cells (SUS'; ACE2 ${ }^{+}$and $\mathrm{SUS}^{+}$: ACE2-) (Fig. 1E). In both cases, the functional distinction of the subpopulation was found to be linked to the key molecular processes related to cellular homeostasis (Fig. 1D-E). Since TMPRSS2 protease has been implicated with the viral entry into the host cell [16], we investigate its expression in all the eight olfactory mucosa cell-types at the single-cell resolution. 
Our results indicate that unlike $A C E 2$, which harbors specialized cell-type-specific expression, detectable levels of TMPRSS2, was observed in a subset of all the investigated cell-types of the human olfactory mucosa, with the prominent expression in the GBC's, Bowman's gland cells, and sustentacular cells (Fig2A-D). Since 2019-nCoV entry into the host cell requires both ACE2 and TMPRSS2, we next investigated the cellular co-occurrence of these two moieties. Our co-expression analysis at the single-cell resolution further revealed the higher propensity of 2019-nCoV into a subset of sustentacular cells ( $\sim 75 \%$ of the total positive cells), in addition to the minor population of Olfactory GBCs and Bowman's gland cells (Fig2E). Taken together, these results collectively suggest that olfactory sensory neurons largely lack the host-specific proteins essential for the cellular entry of 2019-nCoV, whereas supporting and stem cells of the olfactory mucosa are highly susceptible to the viral infection.

\section{Comparable ACE2 expression in the olfactory mucosa in mammals}

Recent studies indicate that the high rate of transmission 2019-nCoV2 is remarkably higher as compared to the related SARs-CoV. It is now being proposed that such a high transmission rate is attributed to genetic recombination events at the $S$ protein of the $\mathrm{RBD}$ region [19]. Although it has been speculated that the 2019-nCoV is transmitted to humans from animal sources [19], little is known about the capability of the other mammal species to act as carriers. Notably, in a recent report, monkeys have been confirmed to have the ability to be carriers of this infection [20]. We asked if other common mammalian species are also at the risk of developing 2019-nCoV mediated loss-of-olfaction. To test this, we estimated the levels of ACE2 and TMPRSS2 transcripts in the bulk transcriptomes, sampled from the olfactory mucosa of 5 mammalian species. Our results indicate that the relative abundance of ACE2 and TMPRSS2 in the olfactory mucosa is comparable in all the tested species (Fig. 1F). These analyses clearly suggest that similar to humans, the olfactory system of other mammals is also at potential risk of $2019-n C o V$ infection. 


\section{Discussion and Future Directions}

In addition to the infection-induced multi-organ dysfunction, a major bottleneck in combating the pandemic outbreak of $2019-\mathrm{nCoV}$ is the availability and accessibility of the diagnostic methods to masses worldwide. Although major improvements have been made in developing 2019-nCoV centric molecular diagnostic kits, their timely availability to the masses across worldwide will require a substantial amount of time and resources. Recently, multiple clinicians indicate the abrupt loss of smell in a large number of 2019-nCoV infected individuals, particularly from Britain, the US, France, South Korea, China, Germany, and Iran, therefore, collectively reinforcing its potential application as the first line of diagnostics in the patients possessing 2019-nCoV hallmark symptoms. Although these symptoms are prevalent in the majority of 2019-nCoV infected individuals, so far the impacted cell type of the olfactory mucosa mediating these phenotypes is still unknown. We report here that loss of smell in the infected patients is most unlikely due to the direct impairment of the olfactory sensory neurons. Moreover, our results indicate that the sustentacular cells, Bowman's cells, and olfactory stem cells are the potential cell types that are highly susceptible to viral entry (Fig. 1 and 2). Although, these cell-types are not sensory in function, but are known to play a crucial role in the maintenance of the olfactory organ [21-24]. Although little is known about the exact role of sustentacular cells in influencing the sensing functionality of the receptor neurons, however, they are known to provide metabolic and physical support to the olfactory mucosa [25]. Similarly, is the case with olfactory stem cells, where their direct or indirect involvement in influencing odorant detection is not reported, however, they are known to play a crucial role in the regeneration of the olfactory epithelium upon lesions $[26,27]$. Due to the potential damage of the reserve olfactory stem cells and the crucial supporting cells upon 2019-nCoV infection, it is most likely that even after recovery, the patients may encounter loss of smell until the cellular composition of the olfactory mucosa reestablishes. Collectively, our study provides the first line of evidence that a subpopulation of olfactory cells is potentially equipped with cell surface receptors which can be exploited by the virus for gaining entry into the cell (Fig. 3). 


\section{Methods}

For the considered single-cell studies, raw read count data were downloaded from GEO (GSE139522). We performed the majority of our analyses including cell/gene filtering, clustering and differential expression analysis using the widely used Seurat software suite [18]. Inbuilt functions NormalizeData(), FindVariableFeatures(), ScaleData(), RunPCA(), DimPlot(), FindNeighbors() and FindClusters() were used for the various standard steps of single-cell expression data analysis. Notably, annotation of the cell clusters was performed using the set of markers indicated in the original study (Fig. S1) [17]. R script used for the analysis can be found at shorturl.at/PT245.

Uniformly processed bulk RNA sequencing data possessing transcriptomic profiles of whole olfactory mucosa from 5 mammalian species i.e. human, monkey, marmoset, mouse, and rat were downloaded from a recent publication from Saraiva and colleagues [28]. FPKM values were log-transformed (base=2) and used for plotting the bar charts. The student's t-test was used to calculate the differences in the mean values between all species. A p-value $<0.05,<0.01$ and $<0.001$ is denoted as $\left({ }^{*}\right),\left({ }^{* *}\right)$, and $\left({ }^{* * *}\right)$, respectively.

\section{Figure Legend}

Figure 1: Specialized expression of ACE2 in a subpopulation of sustentacular and horizontal basal cells of olfactory mucosa

(A) UMAP based embedding of single-cell expression profiles represents the distinct cell types of the olfactory mucosa, segregated-based on their transcriptional profiles. Cells within a cluster represent similar cell types. (B) UMAP based embedding portrays the relative expression of $A C E 2$ transcript in the indicated olfactory mucosa cell types. (C) Percentage bar graphs representing the relative proportion of ACE2 expressing cells in different cell types. (D-E) Metascape analysis of the differentially expressed genes between ACE2-positive and ACE2-negative cells revealing the prominent 
functional ontologies segregating SUSs and HBCs cellular populations. (F) Bar graph depicting the relative abundance of ACE2 transcript in the olfactory mucosa of 5 indicated mammalian species.

Figure 2: (A) TSNE (t-distributed stochastic neighbor embedding) based embedding of single-cell expression profiles represents the distinct cell types of the olfactory mucosa, segregated-based on their transcriptional profiles. Cells within a cluster represent similar cell types. (B) UMAP based embedding portrays the relative expression of TMPRSS2 transcript in the indicated olfactory mucosa cell types. (C) Bubble plot representing the relative expression and the proportion of TMPRSS2 expressing cells in different cell types. (D) Percentage bar graphs representing the relative proportion of TMPRSS2 expressing cells in indicated cell types. (E) Percentage bar graphs representing the relative proportion of cells co-expressing ACE2 and TMPRSS2 in indicated cell types. (F) Bar graph depicting the relative abundance of TMPRSS2 transcript in the olfactory mucosa of 5 indicated mammalian species.

Figure 3: Graphical representation of the key findings.

\section{Supplementary Figure Legend}

Figure S1: Annotation of the cell clustering using known bonafide cell-type-specific markers.

Heatmap map depicting the relative enrichment of bonafide markers in the indicated cell types of the olfactory mucosa. Scale bar represents the normalized expression values.

Figure S2: Expression of bonafide markers of distinct cell types of the human olfactory mucosa

UMAP based embedding of single-cell expression profiles represents the relative expression of bonafide markers of the distinct human olfactory mucosa cell-types. 


\section{Acknowledgments}

The Ahuja lab is supported by the Intramural Start-up grant from Indraprastha Institute of Information Technology-Delhi (IIIT-Delhi) and Ramalingaswami Re-entry Fellowship, a re-entry scheme of the Department of Biotechnology (DBT), Ministry of Science \& Technology, Govt. of India. The Sengupta lab is funded by the INSPIRE faculty grant from the Department of Science \& Technology (DST), India. We would like to thank the IT-HelpDesk team of IIIT-Delhi for providing assistance with the computational resources. We thank all the members of the Ahuja lab for intellectual contributions at various stages of this project.

\section{Competing interests}

The authors declare that they have no competing interests.

\section{Contributions}

The study was conceived by GA and DS. Experimental workflows were designed by GA, DS, TM, and performed by KG, SKM, SK, AM. Clinical insights were provided by JA. Illustrations were drafted by GA and AM. GA and DS wrote the paper. All authors have read and approved the manuscript.

\section{Bibliography}

1. Khot WY, Nadkar MY. The 2019 Novel Coronavirus Outbreak - A Global Threat. J. Assoc. Physicians India 2020; 68:67-71

2. Cascella M, Rajnik M, Cuomo A, et al. Features, Evaluation and Treatment Coronavirus (COVID-19). StatPearls 2020;

3. Lewis D. Coronavirus outbreak: what's next? Nature 2020; 578:15-16

4. Fadel M, Salomon J, Descatha A. Coronavirus outbreak: the role of companies in preparedness and responses. Lancet Public Health 2020;

5. Lu R, Zhao X, Li J, et al. Genomic characterisation and epidemiology of 2019 novel 
coronavirus: implications for virus origins and receptor binding. Lancet 2020; 395:565-574

6. Paraskevis D, Kostaki EG, Magiorkinis G, et al. Full-genome evolutionary analysis of the novel corona virus (2019-nCoV) rejects the hypothesis of emergence as a result of a recent recombination event. Infect. Genet. Evol. 2020; 79:104212

7. Chen Y, Liu Q, Guo D. Emerging coronaviruses: Genome structure, replication, and pathogenesis. J. Med. Virol. 2020; 92:418-423

8. Chan JF-W, Kok K-H, Zhu Z, et al. Genomic characterization of the 2019 novel human-pathogenic coronavirus isolated from a patient with atypical pneumonia after visiting Wuhan. Emerg. Microbes Infect. 2020; 9:221-236

9. Li W, Moore MJ, Vasilieva N, et al. Angiotensin-converting enzyme 2 is a functional receptor for the SARS coronavirus. Nature 2003; 426:450-454

10. Hofmann H, Pyrc K, van der Hoek L, et al. Human coronavirus NL63 employs the severe acute respiratory syndrome coronavirus receptor for cellular entry. Proc Natl Acad Sci USA 2005; 102:7988-7993

11. Zhang $\mathrm{H}$, Penninger JM, Li Y, et al. Angiotensin-converting enzyme 2 (ACE2) as a SARS-CoV-2 receptor: molecular mechanisms and potential therapeutic target. Intensive Care Med. 2020; 46:586-590

12. Zou X, Chen K, Zou J, et al. Single-cell RNA-seq data analysis on the receptor ACE2 expression reveals the potential risk of different human organs vulnerable to 2019-nCoV infection. Front. Med. 2020;

13. Zhao $Y$, Zhao Z, Wang $Y$, et al. Single-cell RNA expression profiling of ACE2, the putative receptor of Wuhan 2019-nCov. BioRxiv 2020;

14. Zhang $H$, Kang $Z$, Gong $H$, et al. The digestive system is a potential route of 2019-nCov infection: a bioinformatics analysis based on single-cell transcriptomes. BioRxiv 2020;

15. Chai $\mathrm{X}$, Hu L, Zhang $\mathrm{Y}$, et al. Specific ACE2 Expression in Cholangiocytes May Cause Liver Damage After 2019-nCoV Infection. BioRxiv 2020;

16. Hoffmann M, Kleine-Weber H, Schroeder S, et al. SARS-CoV-2 Cell Entry Depends 
on ACE2 and TMPRSS2 and Is Blocked by a Clinically Proven Protease Inhibitor. Cell 2020;

17. Durante MA, Kurtenbach S, Sargi ZB, et al. Single-cell analysis of olfactory neurogenesis and differentiation in adult humans. Nat. Neurosci. 2020; 23:323-326

18. Stuart T, Butler A, Hoffman $P$, et al. Comprehensive Integration of Single-Cell Data. Cell 2019; 177:1888-1902.e21

19. Wong MC, Javornik Cregeen SJ, Ajami NJ, et al. Evidence of recombination in coronaviruses implicating pangolin origins of nCoV-2019. BioRxiv 2020;

20. . Infection with Novel Coronavirus (SARS-CoV-2) Causes Pneumonia in the Rhesus Macaques. 2020;

21. Huard JM, Youngentob SL, Goldstein BJ, et al. Adult olfactory epithelium contains multipotent progenitors that give rise to neurons and non-neural cells. J. Comp. Neurol. 1998; 400:469-486

22. Carr VM, Farbman Al, Colletti LM, et al. Identification of a new non-neuronal cell type in rat olfactory epithelium. Neuroscience 1991; 45:433-449

23. Leung CT, Coulombe PA, Reed RR. Contribution of olfactory neural stem cells to tissue maintenance and regeneration. Nat. Neurosci. 2007; 10:720-726

24. Moran DT, Rowley JC, Jafek BW, et al. The fine structure of the olfactory mucosa in man. J. Neurocytol. 1982; 11:721-746

25. Getchell ML, Getchell TV. Fine structural aspects of secretion and extrinsic innervation in the olfactory mucosa. Microsc. Res. Tech. 1992; 23:111-127

26. Joiner AM, Green WW, Mclntyre JC, et al. Primary cilia on horizontal basal cells regulate regeneration of the olfactory epithelium. J. Neurosci. 2015; 35:13761-13772

27. Iwai N, Zhou Z, Roop DR, et al. Horizontal basal cells are multipotent progenitors in normal and injured adult olfactory epithelium. Stem Cells 2008; 26:1298-1306

28. Saraiva LR, Riveros-McKay $F$, Mezzavilla $M$, et al. A transcriptomic atlas of mammalian olfactory mucosae reveals an evolutionary influence on food odor detection in humans. Sci. Adv. 2019; 5:eaax0396 
A Human Olfactory Mucosa B

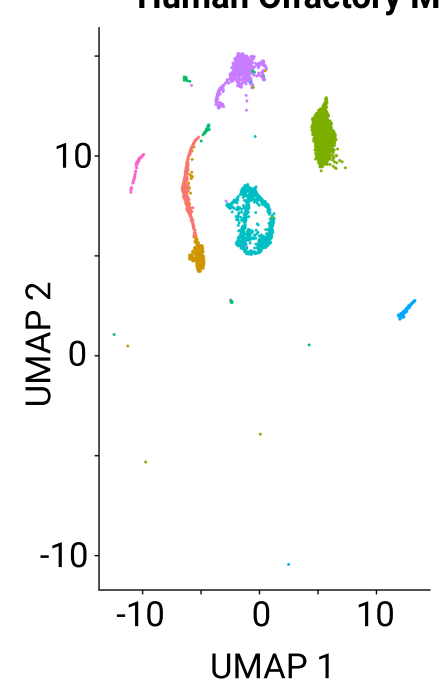

D

HBC+:ACE2+ vs HBC+:ACE2-

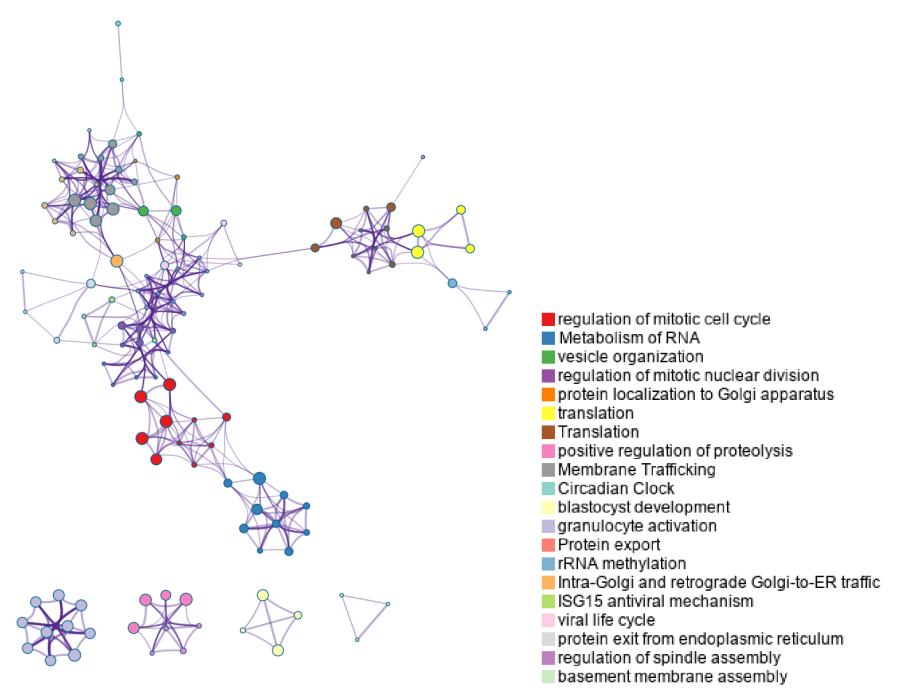

E
C

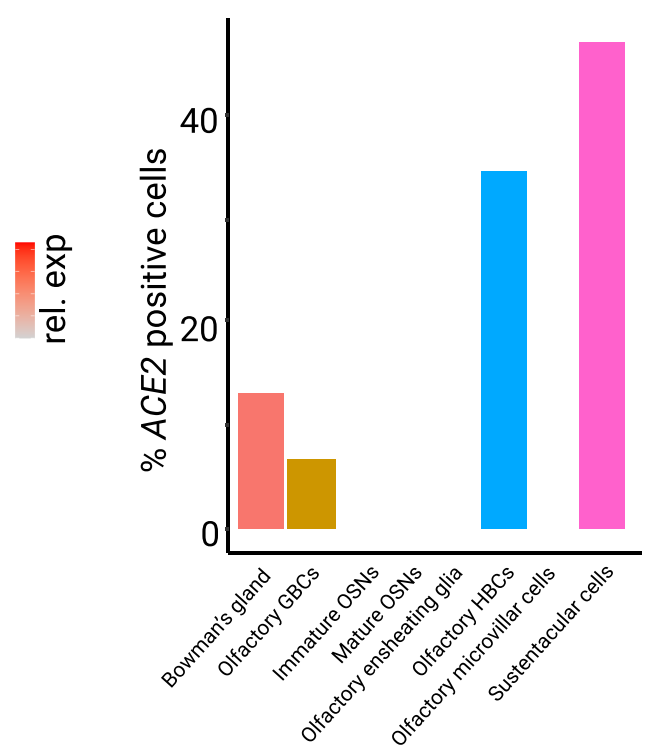

$\mathbf{F}$

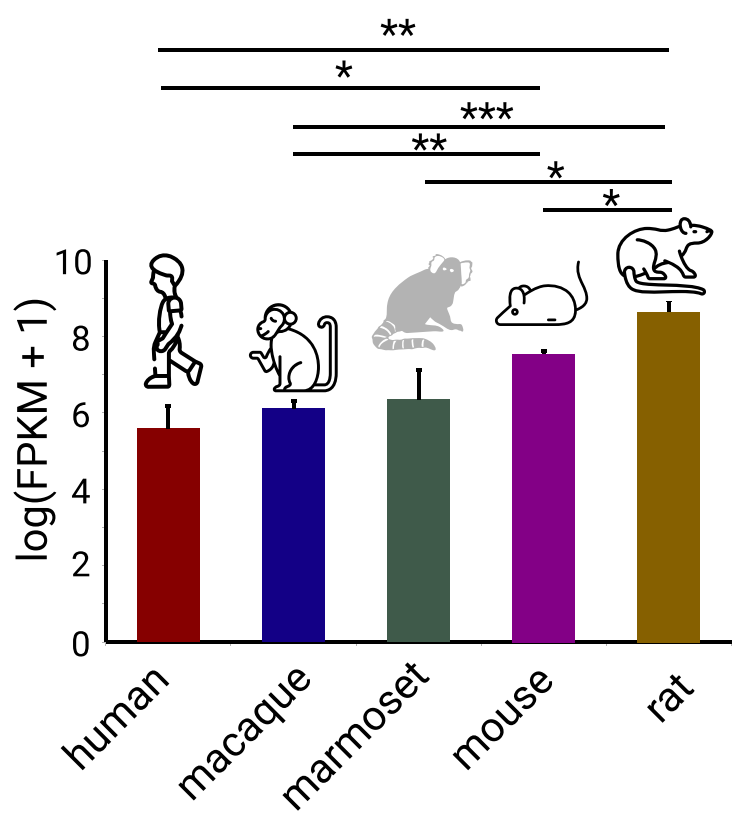

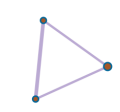
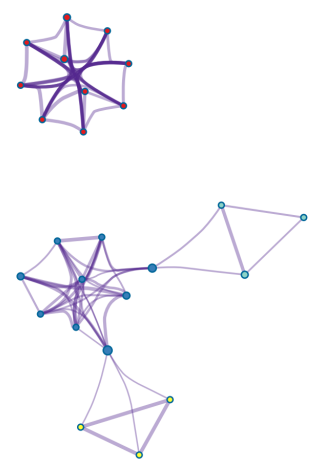

Daerobic respiration cytoplasmic translational initiation C complex spliceosome regulation of filopodium assembly Formation of TC-NER Pre-Incision Complex intracellular protein transmembrane transport - PID P75 NTR PATHWAY PID P53 DOWNSTREAM PATHWAY cellular protein complex disassembly
Translocation of SLC2A4 (GLUT4) to the plasma me Translocation of SLC2A4 (GLUT4) to response to axon injury 
A

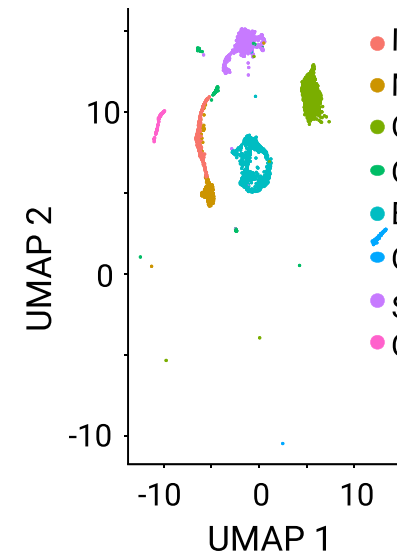

D

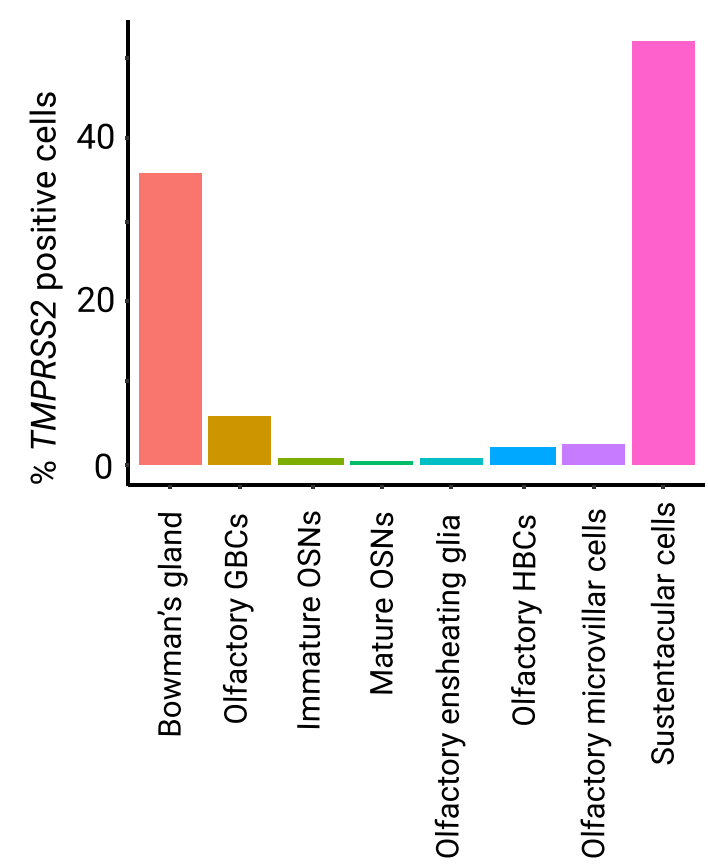

B

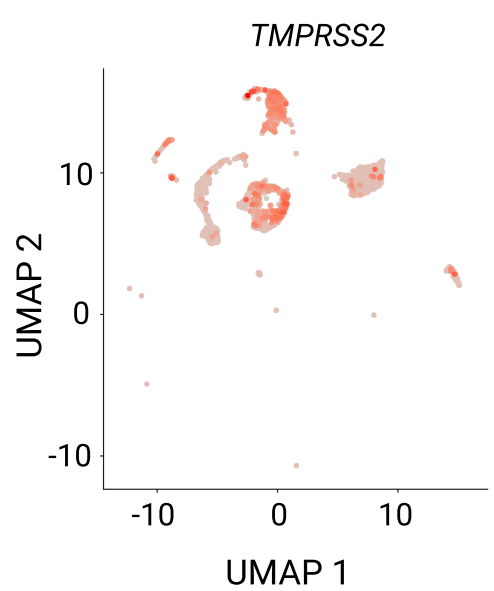

E

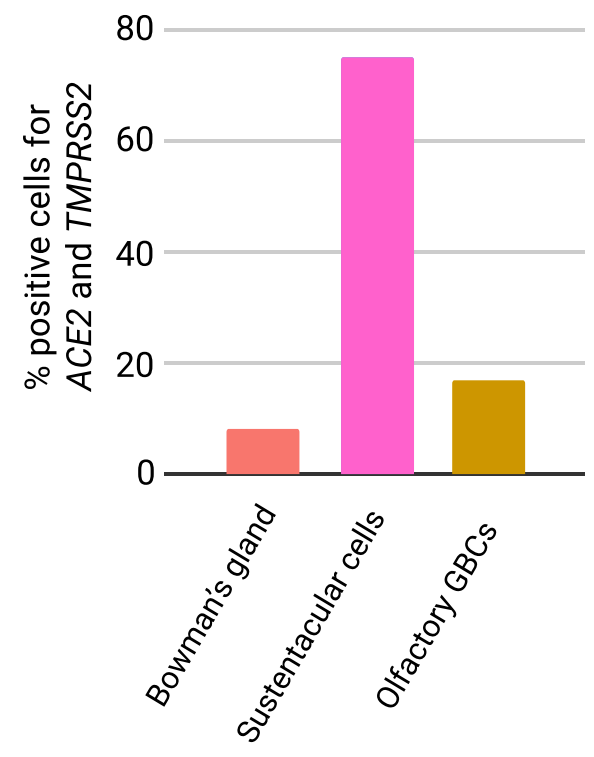

C

Olfactory ensheating glia

Bowman's gland

Olfactory HBCs

Mature OSNs

Immature OSNs

$\begin{array}{lr}: & 0 \\ \circ & 20 \\ \bigcirc & 40 \\ \bigcirc & \end{array}$

F
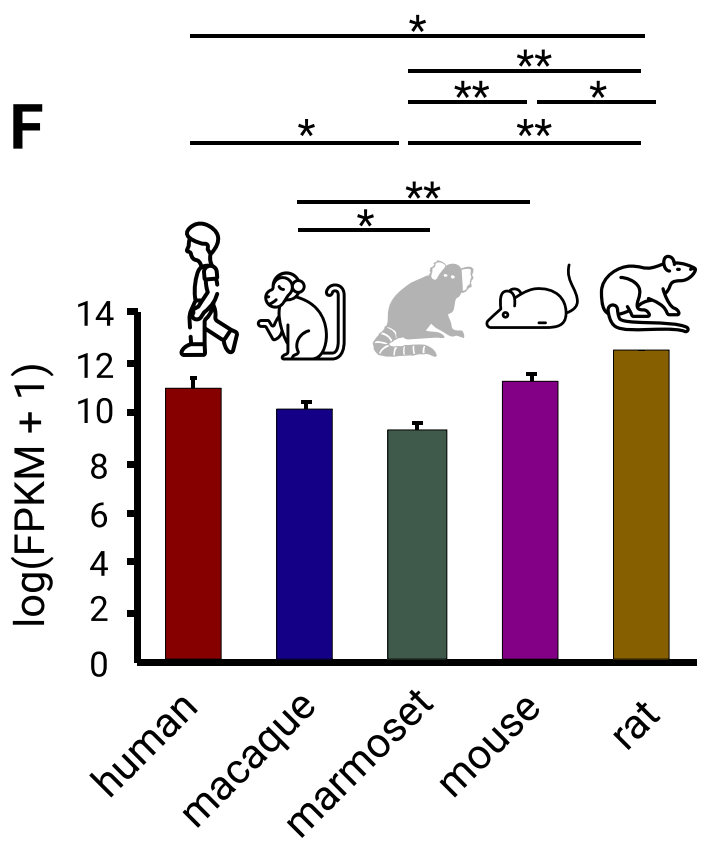


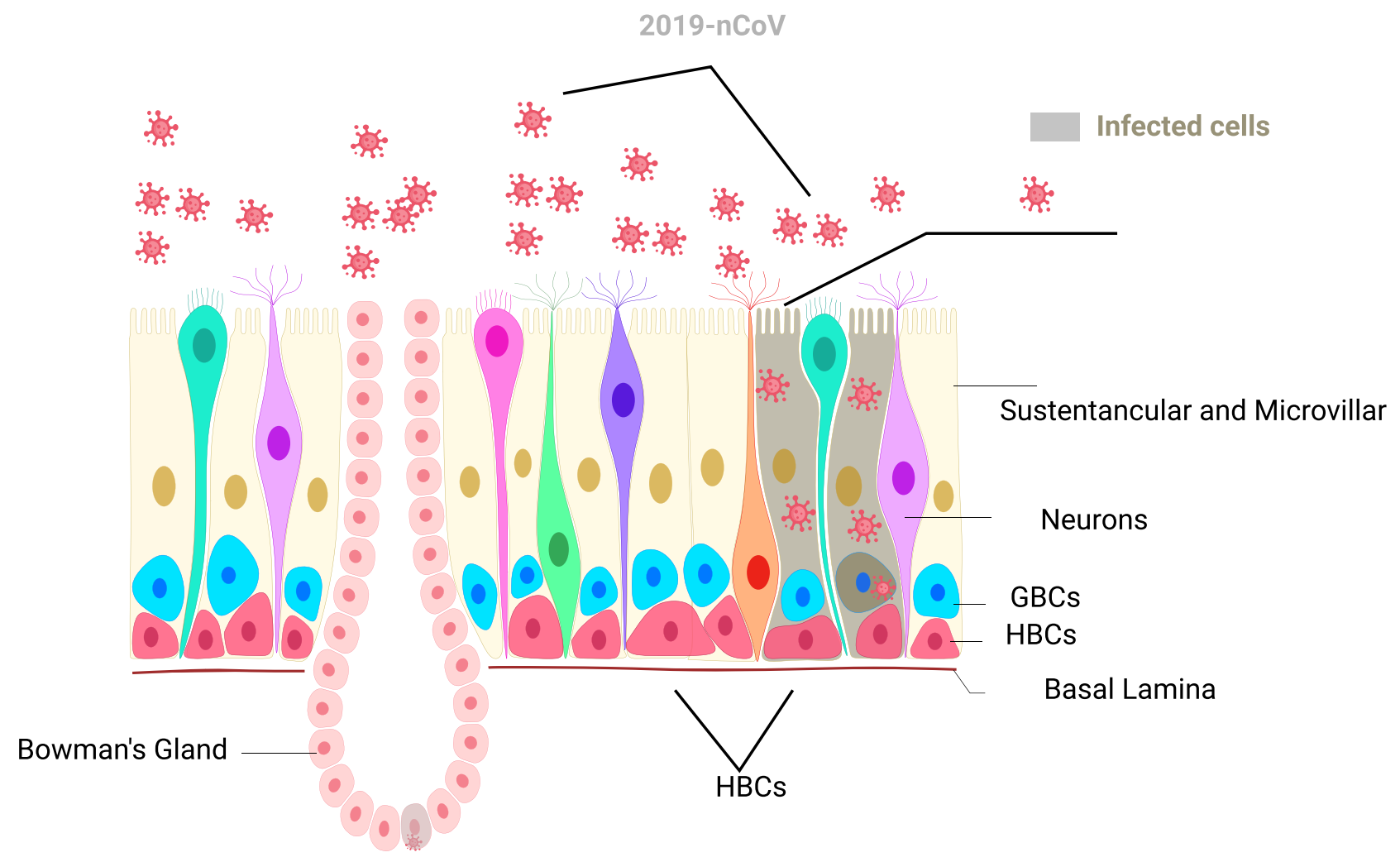




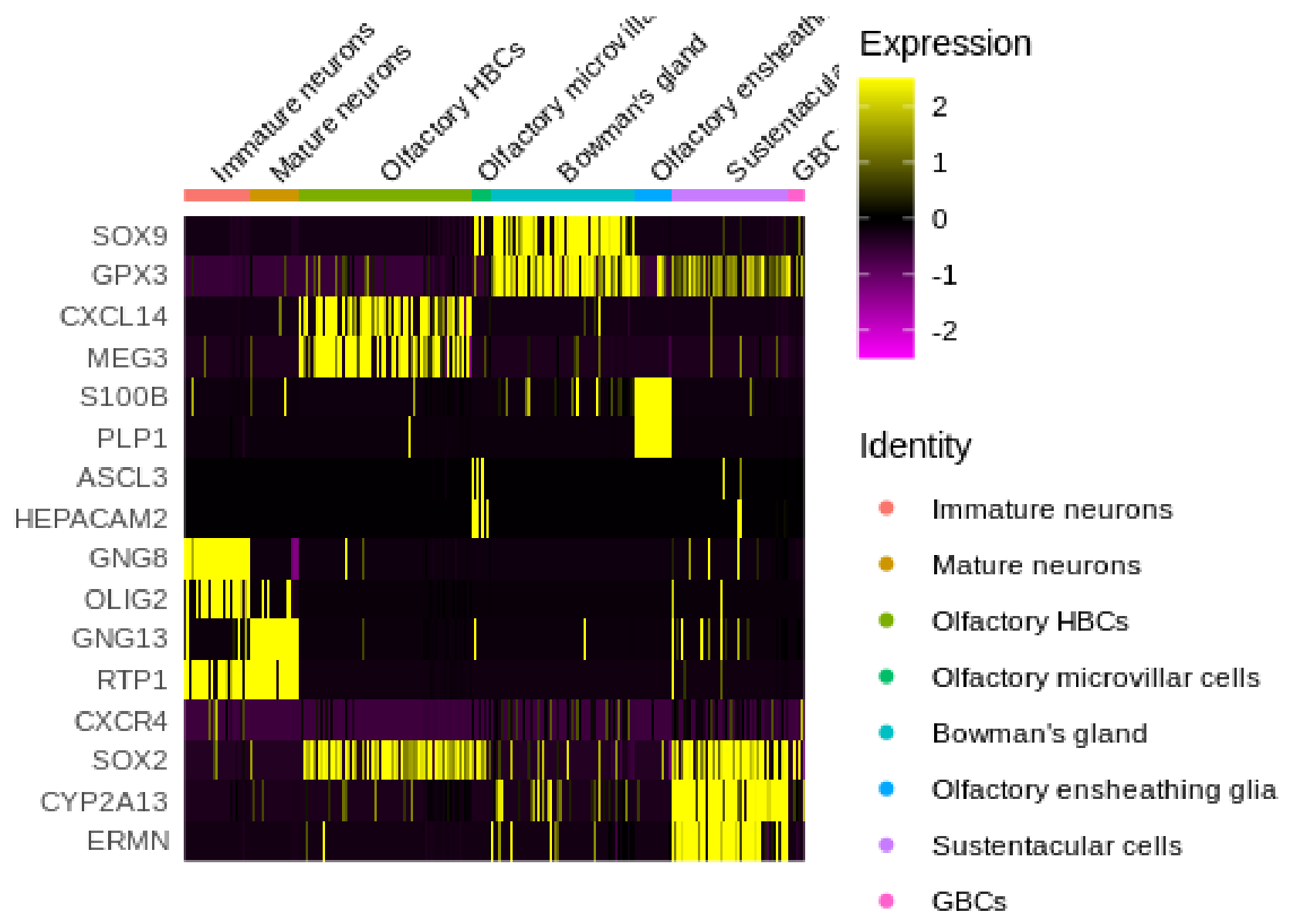

Figure S1 


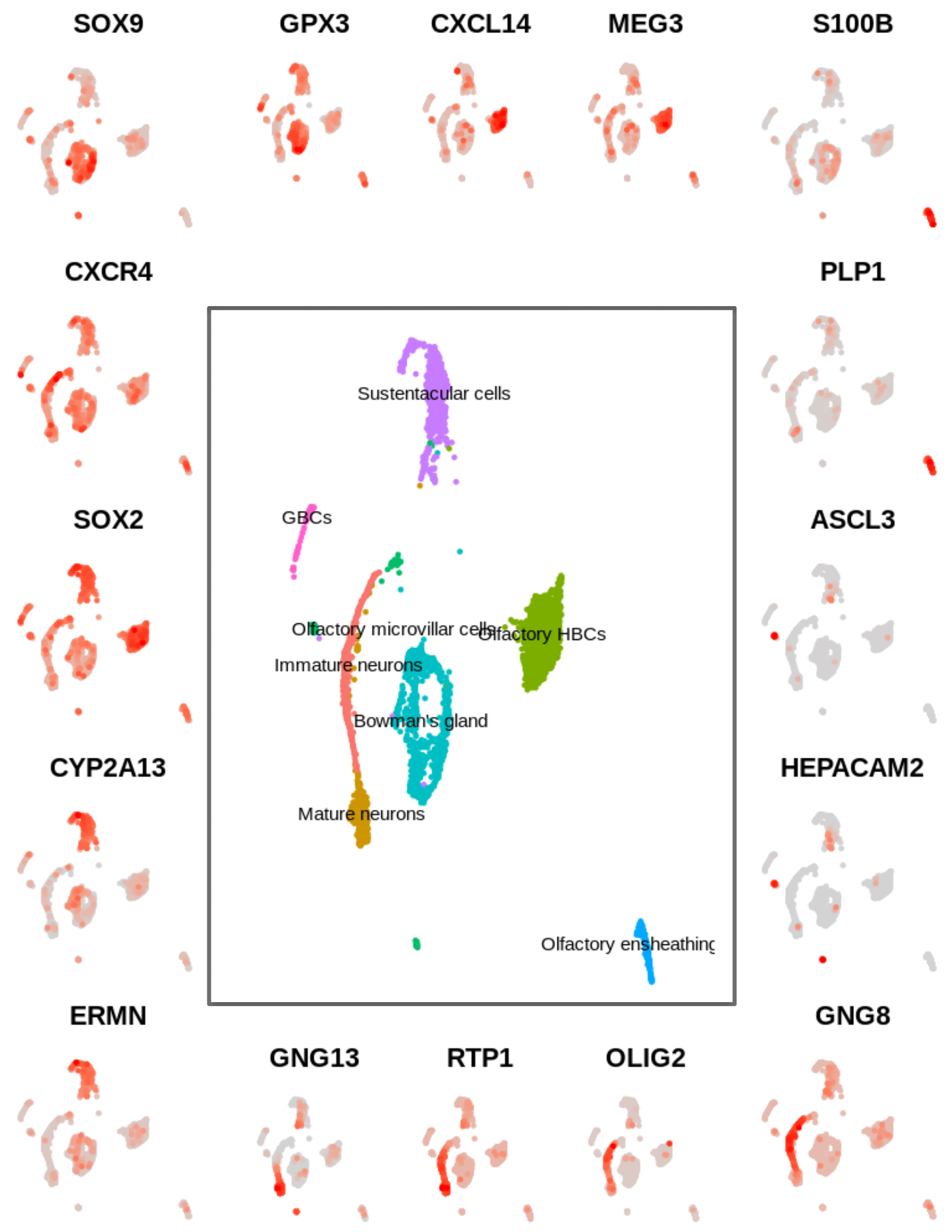

Figure S2 